\title{
Loss of Sun2 promotes the progression of prostate cancer by regulating fatty acid oxidation
}

\author{
Cheng Yajun ${ }^{1, *}$, Ye Chen ${ }^{2, *}$, Li Xiaosa ${ }^{3, *}$, Wang Xiao ${ }^{3}$, Chen Jia ${ }^{3}$, Wang Zhong ${ }^{1}$ and Xu Bin ${ }^{1}$ \\ ${ }^{1}$ Department of Urology, Shanghai Ninth People's Hospital, Shanghai Jiaotong University School of Medicine, Shanghai, China \\ ${ }^{2}$ Department of Urology, Changhai Hospital, Second Military Medical University, Shanghai, China \\ ${ }^{3}$ School of Life Science and Technology, ShanghaiTech University, Shanghai, China \\ *These authors contributed equally to this work
}

Correspondence to: Xu Bin, email: chxb2004@126.com

Wang Zhong, email: zhongwang2000@sina.com

Chen Jia, email: chenjia@shanghaitech.edu.cn

Keywords: prostate cancer, fatty acid oxidation, Sun2, SAA1, overall survival

Received: April 18, 2017 Accepted: June 28, $2017 \quad$ Published: July 12, 2017

Copyright: Yajun et al. This is an open-access article distributed under the terms of the Creative Commons Attribution License 3.0 (CC BY 3.0 ), which permits unrestricted use, distribution, and reproduction in any medium, provided the original author and source are credited.

\section{ABSTRACT}

The role of Sun2 has been described by previous studies in various types of cancers, including breast cancer and lung cancer. However, its role and potential molecular mechanism in the progression of prostate cancer have not been fully elucidated. In the present study, we found that Sun2 expression was reduced in prostate cancer tissues compared with paired normal tissues, and that low expression of Sun2 was significantly correlated with Higher Gleason scores, postoperative T stage (pT), Lymph nodal invasion and Clinical pathological stages. In addition, reduced Sun2 Expression predicts poor survival of prostate cancer patients and could serve as an independent predictor of prostate cancer patients overall survival (OS).Furthermore, Sun 2 overexpression inhibits the prostate cancer cells growth, and Sun 2 knockdown promotes the prostate cancer cells growth both in vitro and vivo. Mechanical silencing of , Sun2 promoted fatty acid oxidation (FAO) in prostate cancer, prostate cancer cells growth promoted by Sun2 silencing could be reversed by the FAO inhibitor Etomoxir. Additionally, we also showed that serum amyloid A1 (SAA1) play a vital role in FAO, ATP and cell growth promoted by Sun 2 loss in prostate cancer. These results suggest that Loss of Sun 2 promoted the prostate cancer progression by regulating FAO.

\section{INTRODUCTION}

Cancer is the leading cause of death since 2010 and one of the major public health problem in china [1]. With advances in biopsy technology and increasing incidence of prostate-specific antigen screening or others factors, more cases of prostate cancer have been diagnosedin past decade [2]. Although traditional therapies, including surgical resection, endocrinotherapy and chemotherapyare still effective therapies for prostate cancer, transitions of prostate cancer to castration-resistant prostate cancer (CRPC) remains a lethal disease and kills thousands of men every day [3]. For men, it is most frequently diagnosed cancer and the second leading cause of cancerdeath in western countries [4]. Serum prostate-specific antigen (PSA), as a Conventional prognostic factor, is already recognized as available biomarker for diagnosis and prognosis of prostate cancer. Numerousstudies have demonstrated that the prostate cancer patients with equivalent PSA level may have different clinical outcomes [5]. As prostate cancer is a heterogeneous multifocal disease with highly variable natural history, with respect to Age, race, geographic location, familial history and genetic background, [6], it is difficult to predict its initiation, progression and prognosis of prostate cancer, and it need us to find new biomarkers of prostate cancer to diagnose and predict clinical outcome [9-11].Therefore, it need to identify specific biomarkers for diagnosis and novel target for therapy to improve prognosis and treatment of this cancer. 
Nuclear envelope (NE) separates chromosomes from the cytoplasm in eukaryotic cells and NE proteins play a vital role in various biological effects by mediating genome organization, gene expression and mutatation [7]. The NE is a double lipid bilayer, comprising outer (ONM) and inner nuclear membranes (INM) that form the perinuclear space (PNS) [8]. The LINC (linker of nucleoskeleton and cytoskeleton) complex connects the nucleus with the cytoskeleton by assembling NE proteins. It consists of two kinds of protein families: nesprin protein and SUN protein, which was located in the outer and inner nuclear membrane respectively, interacting each other in PNS [9]. Additionally, SUN protein, consisting of Sun1 and Sun2, also interacts with lamins, functioning as nuclear skeleton in the nucleoplasm. Dysregulation of Sun2, as a characteristic NE protein, is associated with many human diseases, including cancers. Overexpression of Sun2 could prevent HIV infection by interfering with viral replication [10]. Recent studies have shown that SUN2 inhibit a variety of tumor malignancies in vitro and in vivo. Heish et al [11] reported that Sun2 was down-expressed in the central nervous system (CNS) embryonal tumors asa novel tumor suppressor. And miR$221 / 222$ promoted the central nervous system embryonal tumorigenesis bytargeting Sun2. Sun2 expression was also decreased in breast cancer and may play a tumor suppressor role [12]. Furthermore, Xiao-bin et al [13] demonstrated that downregulation of Sun2 promoted lung cancer progression by regulating the Warburg effect. However, expression and role of SUN2 in prostate cancer is unkown and needs to be explored.

To discover new therapeutic targets for prostate cancer, it is essential for exploring the molecular mechanisms underlyingtumor progression. The aim of the present study was to examine the relationship between Sun2 expression and clinicopathologic outcomes by tissue microarray (TMA) of 90 prostate cancer patients, and then investigate the role of Sun2 in prostate cancer cell growth in vitro and in vivo. In addition, the impact of Sun 2 on the metabolism of prostate cancer cells was also evaluated. Finally, RNA sequence was used to explore the potential mechanism of prostate cancer progression inhibited by Sun2.

\section{RESULTS}

\section{Sun2 is downregulated in prostate cancer and correlates with clinicopathological parameters}

To examine whether the Sun2 protein was differentially expressed in prostate cancer tissues vs.normal tissues, immunohistochemical staining and qRT-PCR were performed. As shown in Figure 1A, Sun2 protein was mainly localized in the nucleus. The results of staining scoring in the 90 prostate cancer tissues and adjacent benign tissues showed that Sun2 expression was downregulated in prostate cancer $(61 / 90,67.5 \%)(7.24 \pm 4.42$ vs. $3.86 \pm 3.3$,
$P<0.05$ ) (Figure 1B). Furthermore, Sun2 mRNA expression was also downregulated $(26 / 35,74.3 \%)$ in prostate cancer patients $(P<0.05)$ (Figure 1C). We further analyzed the relationship between the expression levels of Sun2 and several clinicopathological features of prostate cancer patients, and found that low expression of Sun2 was significantly correlated with Gleason score $(P=0.018)$, Postoperative T stage (pT) $(P=0.03)$, Lymph nodal invasion $(P=0.004)$ and clinical pathological stage $(P=0.012)$ (Table 1). No significant association was seen between age, PSA at diagnosis and Sun2 expression (Table 1).

\section{Decreased Sun2 expression predicts poor survival in prostate cancer and is an independent predictor of OS}

To assess the relationship between Sun2 expression and the prognosis of prostate cancer patient, Kaplan-Meier survival analysis was performed and the results showed that low Sun2 expression predicted poor survival of prostate cancer patients $(P<0.01)$ (Figure 2$)$. Meanwhile, follow-up time was no significantly correlated with high or low Sun2 expression ( $\mathrm{m} \pm \mathrm{sd}, 85.4 \pm 23.6$ vs. $89.6 \pm 32.4$, $P>0.05)$. Moreover, univariate analysis showed that Sun2 expression $(P<0.01)$, Gleason score $(P=0.02)$, Lymph nodal invasion $(P<0.01)$ and TNM Stage $(P<0.01)$ were the factors affecting the survival rate in postoperative prostate cancer patients (Table 2). Next, multivariate analysis was performed and the results showed that low Sun2 expression $(P<0.01)$ and TNM stage $(P=0.02)$ were the independent factors predicting the prognosis of prostate cancer patients.

\section{Sun2 overexpression inhibits the prostate cancer cell growth}

In order to determine the role of Sun2 in prostate cancer, CRPC cell line pc-3 with relatively low expression Sun2 was transfected with Sun2. As shown in Figure 3A, Sun2 was overexpressed in PC-3 cells by western blot identification, and a stable line was established. Furthermore, we found that Sun2 overexpression inhibited the cancer cell proliferation by CCK-8 evaluation at 48 and $72 \mathrm{~h}(P<0.05)$ (Figure 3B). Moreover, cell apoptosis and the cell cycle induced by Sun 2 overexpression were assessed by flow cytometry. The results showed that Sun2 overexpression promoted the cancer cell apoptosis $(P<0.05)$ (Figure 3C) and induced cell cycle arrest in G1/S phase $(P<0.05)$ (Figure 3D) compared with the control.Finally, a xenograft tumor model was conducted to evaluate the growth of prostate cancer induced by Sun2. It was found that the mean tumor weight was significantly reduced in Sun2 overexpression tumors compared with vector control tumors $(p<0.05)$ (Figure 3E). The above results suggest that Sun2 inhibited the progression of prostate cancer. 


\section{Sun2 silencing promotes the prostate cancer cell growth}

CRPC cell line C4-2 with relatively high Sun2 expression was transfected with sh-Sun2. Sun2 protein expression after shRNA infection was determined by immunoblotting analysis (Figure 4A), showing that it was significantly decreased compared with control. The effect of Sun2 knockdown on rostate cancer cells proliferation was evaluated using CCK-8 kit. Cpt1c significantly inhibited cell growth in Sun2-shRNA transfected cells compared with the control at 48 and $72 \mathrm{~h}$ $(P<0.05)$ (Figure 4B). Moreover, we found that Sun2 silencing inhibited the cell apoptosis by flow cytometry analysis. Additionally, the number of cells in $\mathrm{G} 1$ and $\mathrm{S}$ phase $(P<0.05)$ was decreased and the number of cells in G2 phase was increased as compared with control (both $P<0.05$ ) (Figure 4C, 4D).Finally, tumorigenicity assay in nude mice showed that the mean tumor weight was significantly increased in sh-Sun2 tumors as compared
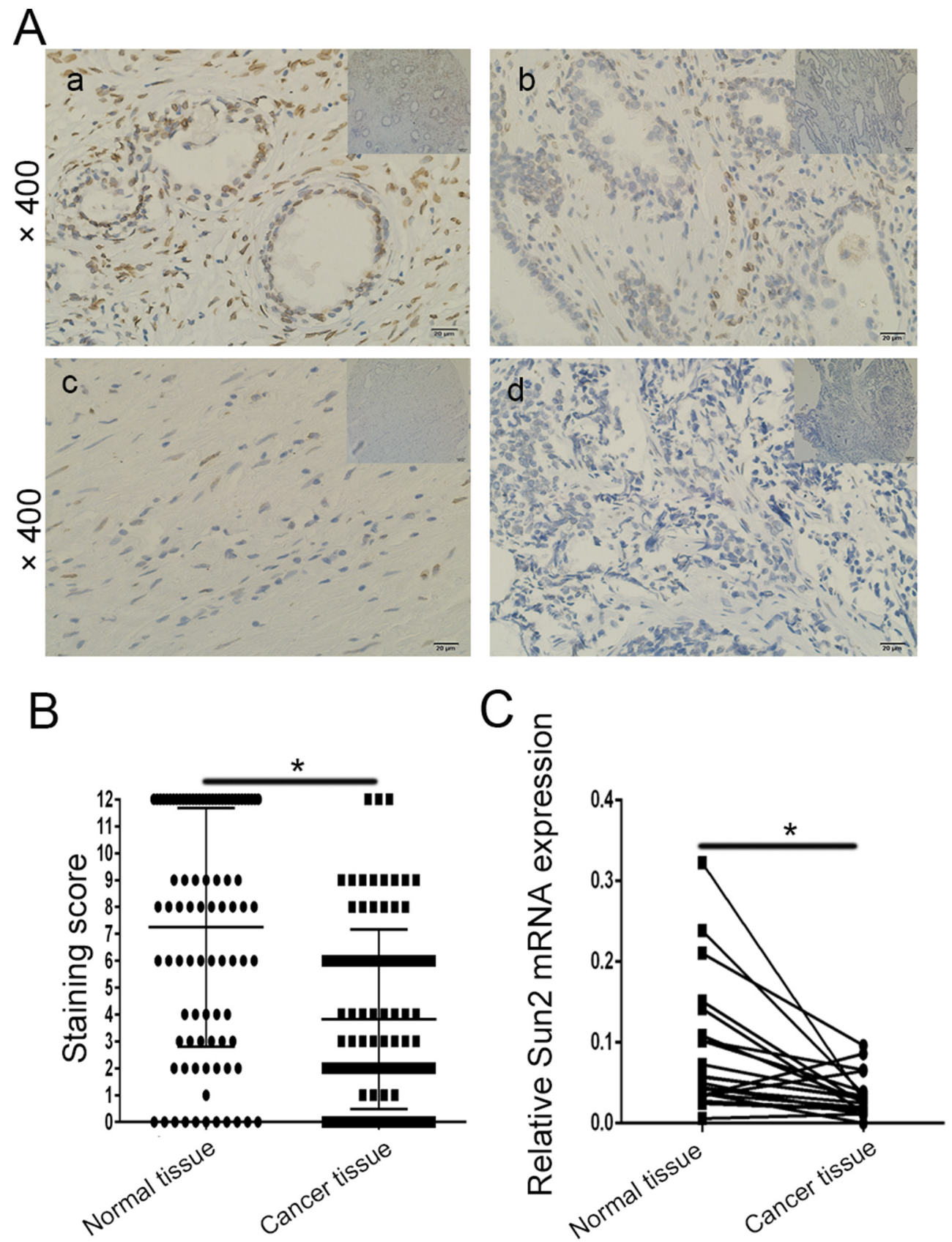

Figure 1: The expression pattern of Sun2 in prostate cancer tissues. (A) a: Normal tissues (positive), b-d: immunohistochemistry staining of Sun2 in prostate cancer samples, b (positive), c (medium), d (weak); (B) The mean staining score of TTP in prostate cancer tumor tissues $v s$.matched adjacent normal tissues $(n=90)$; $(\mathbf{C})$ The mean expression of TTP mRNA in prostate cancer tissues $v s . m a t c h e d$ adjacent normal tissues $(n=35) . * P<0.05, * * P<0.01$. 
Table 1: Correlation between the clinicopathologic variables and Sun2 expression in prostate caner

\begin{tabular}{|c|c|c|c|c|c|}
\hline \multirow{2}{*}{ Clinical character } & \multirow{2}{*}{ Cases } & Sun2 expression & \multirow{2}{*}{ Low } & \multirow{2}{*}{$\chi^{2}$ value } & \multirow{2}{*}{$p$ value } \\
\hline & & High & & & \\
\hline \multicolumn{6}{|l|}{ Age (years) } \\
\hline$<60$ & $31(34.4 \%)$ & $11(35.5 \%)$ & $20(64.5 \%)$ & 0.23 & 0.64 \\
\hline$\geq 60$ & $59(65.6 \%)$ & $18(30.5 \%)$ & $41(69.5 \%)$ & & \\
\hline \multicolumn{6}{|l|}{ Gleason score } \\
\hline$<8$ & $58(64.5 \%)$ & $24(41.3 \%)$ & $34(58.7 \%)$ & 6.26 & $0.018 *$ \\
\hline$\geq 8$ & $32(35.5 \%)$ & $5(15.7 \%)$ & $27(84.3 \%)$ & & \\
\hline \multicolumn{6}{|l|}{ PSA at diagnosis (ng/ml) } \\
\hline$<10$ & $41(45.6 \%)$ & $10(24.4 \%)$ & $31(75.6 \%)$ & 2.11 & 0.178 \\
\hline$\geq 10$ & $49(54.4 \%)$ & $19(38.8 \%)$ & $30(61.2 \%)$ & & \\
\hline \multicolumn{6}{|l|}{ Tumor infiltration } \\
\hline $\mathrm{T} 1-\mathrm{T} 2$ & $50(55.6 \%)$ & $20(33.3 \%)$ & $30(66.7 \%)$ & 5.31 & $0.03 *$ \\
\hline $\mathrm{T} 3-\mathrm{T} 4$ & $40(44.4 \%)$ & $9(66.2 \%)$ & $31(33.8 \%)$ & & \\
\hline \multicolumn{6}{|l|}{ Lymph nodal status } \\
\hline Negative & $48(53.3 \%)$ & $22(45.8 \%)$ & $26(54.2 \%)$ & 8.7 & $0.004 * *$ \\
\hline Positive & $42(46.7 \%)$ & $7(16.7 \%)$ & $35(83.3 \%)$ & & \\
\hline \multicolumn{6}{|c|}{ Clinical pathological stage } \\
\hline$<\mathrm{T} 2 \mathrm{C}$ & $54(60 \%)$ & $23(44.6 \%)$ & $31(55.4 \%)$ & 6.65 & $0.012 *$ \\
\hline$\geq \mathrm{T} 2 \mathrm{C}$ & $36(40 \%)$ & $6(16.7 \%)$ & $30(83.3 \%)$ & & \\
\hline Total & $90(100.0 \%)$ & $29(32.2 \%)$ & $61(67.8 \%)$ & & \\
\hline
\end{tabular}

Statistical analyses were carried out using Pearson $\chi^{2}$ test.

$* P<0.05$ and $* * P<0.01$ were considered significant.

with the control $(p<0.05)$ (Figure 4E). These results suggest that Sun2 silencing promoted the progression of prostate cancer.

\section{Sun2 suppresses the fatty acid oxidation (FAO) in prostate cancer}

Sun2 can decrease the energy production by suppressing aerobic glycolysis of cancer cell as previously described [11], therefore, ATP concentrations was evaluated. Consistent with previous report, silencing Sun2 promoted the ATP production of prostate cancer cells (Figure 5A). Next, glucose uptake and the glycolytic rate was assessed to determine whether Sun2 had any impact on aerobic glycolysis. Our results showed that silencing Sun2 can not increase glucose uptake (data not shown) and the glycolytic rate (Figure 5B) in prostate cancer cells. Recent studies suggest that it relied on lipid oxidation more
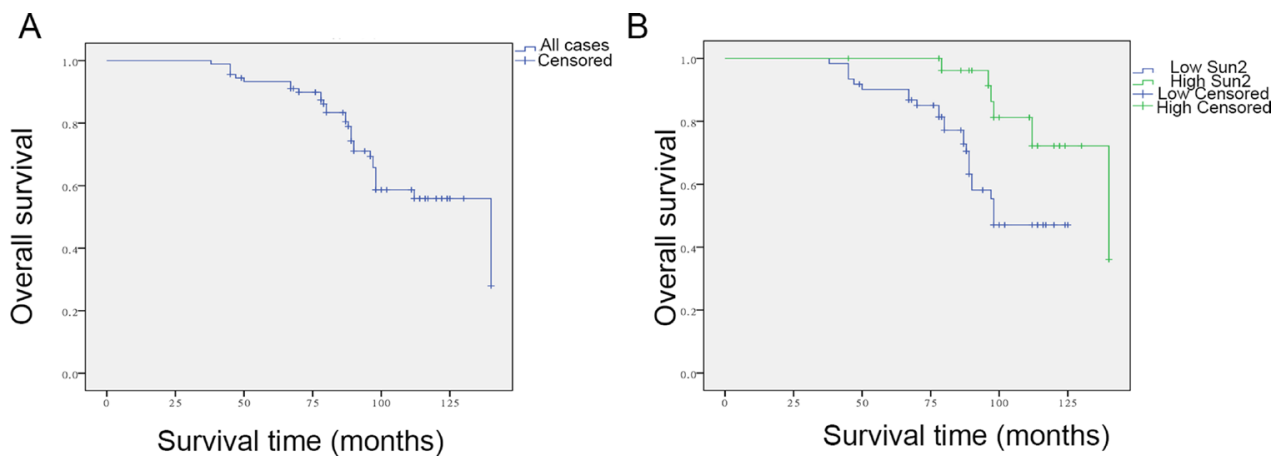

Figure 2: Relationship between low Sun2 expression and outcome of patients with prostate cancer. (A) Kaplan-Meier curves for overall survival (OS) of 90 prostate cancer patients. (B) Kaplan-Meier curves for OS in 90 prostate cancer patients with low or no expression or high expression of Sun $2 . * P<0.05, * * P<0.01$. 
Table 2: Univariate and multivariate analysis of overall survival of prostate cancer patients

\begin{tabular}{lcccccc}
\hline Clinical character & \multicolumn{3}{c}{ Univariate analysis } & \multicolumn{3}{c}{ Multivariate analysis } \\
\hline & HR & $\mathbf{( 9 5 \%}$ CI) & $\boldsymbol{p}$ value & HR & $\mathbf{( 9 5 \%}$ CI) & $\boldsymbol{p}$ value \\
\hline Sun2 expression & 3.84 & $2.1-6.98$ & $<\mathbf{0 . 0 1 * *}$ & 3.01 & $1.61-5.89$ & $<\mathbf{0 . 0 1 * *}$ \\
Age (years) & 1.12 & $0.8-1.54$ & 0.80 & & & \\
Gleason score & 2.34 & $1.44-3.56$ & $\mathbf{0 . 0 2 *}$ & 1.45 & $0.79-2.97$ & 0.28 \\
PSA at diagnosis & 1.43 & $0.87-1.98$ & 0.69 & & & \\
Tumor infiltration & 1.86 & $0.97-2.04$ & 0.06 & & & 0.49 \\
Lymph Nodal status & 2.57 & $1.51-3.61$ & $<\mathbf{0 . 0 1 * *}$ & 1.67 & $0.87-3.04$ & $\mathbf{0 . 0 2 *}$ \\
TNM staging & 3.25 & $1.97-5.15$ & $<\mathbf{0 . 0 1 * *}$ & 2.83 & $1.42-4.34$ & \\
\hline
\end{tabular}

than on aerobic glycolysis to fulfill the energetic demands in prostate cancer [12]. Therefore, FAO was measured by Cellular OCR. We found that silencing Sun2 facilitated the FAO as compared with the control (Figure 5C). And FAO enhanced by low Sun2 expression could be downregulated by a FAO inhibiter ETO in a concentration- dependent manner (Figure 5D). Due to FAO facilitated by Sun2 silencingcould be neutralized by ETO, with $10 \mu \mathrm{mol}$ concentration, it was selected to culture with sh-Sun2 cell lines. The Results showed that growth of prostate cancer cells promoted by low Sun2 expression can be reversed by ETO at $10 \mu \mathrm{mol}$ concentration (Figure 5E). These results suggest that low Sun2 promoted the prostate cancer progression by enhancing FAO rather aerobic glycolysis.
A

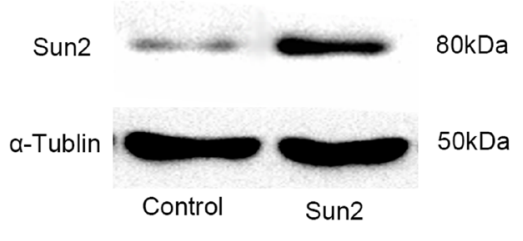

C

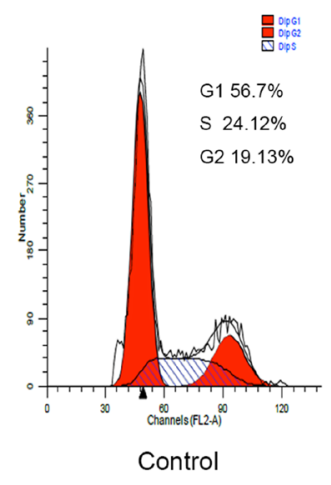

E

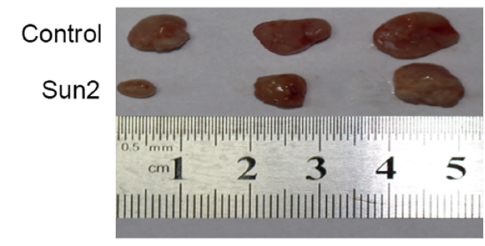

B

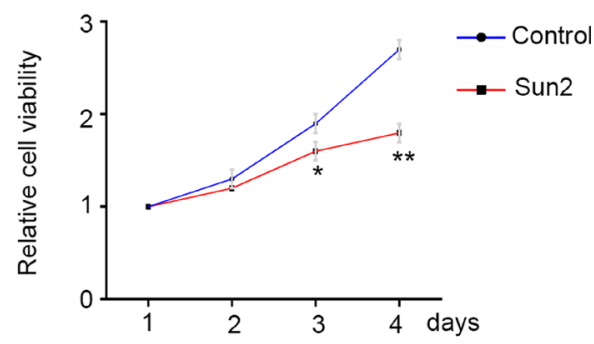

D
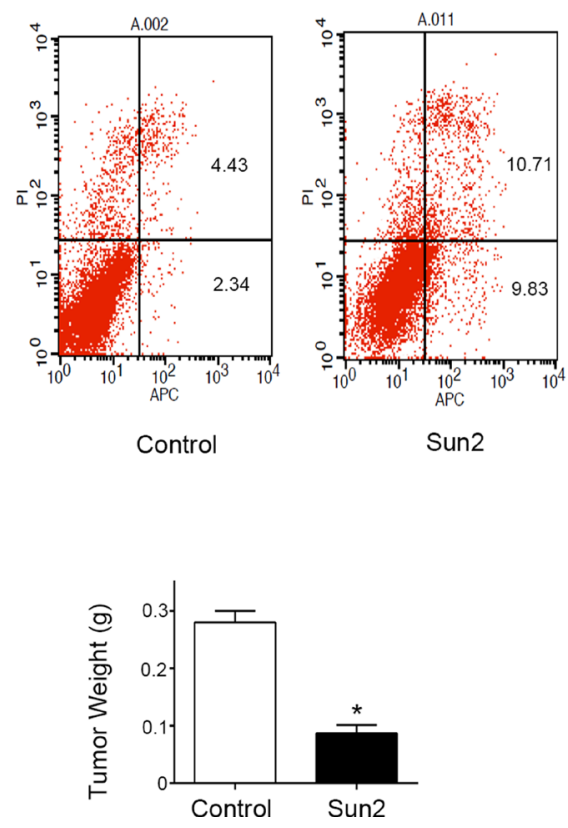

Figure 3: Sun2 overexpression inhibits prostate cancer growth. (A) Western blot was used to confirm the Sun2 transfection efficiency. (B) Cell proliferation levels were measured using the cell counting kit-8 at 1,2,3 and 4 days. (C) and (D) Cell-cycle distribution and cell apoptosis were analyzed by flow cytometry, respectively. (E) Tumorigenicity assay in nude mice was performed in Sun2 overexpression stable cell lines $v$ s. control. $* P<0.05, * * P<0.01$. 


\section{The potential mechanism of FAO regulated by Sun 2 in prostate camcer}

RNA Sequence analysis of sh-Sun2 and control prostate cancer cells was performed to explore mechanism of FAO regulated by Sun2 in prostate cancer. Serum amyloid A1 (SAA1), serum amyloid A2 (SAA2), receptor tyrosine kinase (AXL), Ras-related protein Rab-1A (RAB1A) and HMCES were identified (Figure 6A). Recent reports showed that SAA played an important role in lipid metabolism [14]. And we found that overexpression or knockdown of Sun2 decreased or increased SAA1 expression, respectively, in prostate cancer cells (Figure 6B). Next, we observed a significant inverse correlation between Sun2 and SAA1 in prostate cancer tissues $(P=0.0019)$ (Figure 6C). In addition,
SAA1 was highly overexpressed in prostate cancer tissues as compared with normal tissues $(P<0.05)$ (Figure 6D). These results suggested that SAA1 may playan import role in prostate cancer progression regulated by Sun2. sh-Sun2 stable cell lines were transfected with $\mathrm{Si}$ SAA1 and the transfection efficiency was determined by immunoblotting analysis, showing SAA1 expression was significantly decreased in sh-Sun2 stable cell lines were transfected with Si-SAA1 as compared with shSun2 stable cell lines (Figure 6E). FAO assay showed that Si-SAA1 partially suppressed the FAO in sh-Sun2 state cell lines $(P<0.05)$ (Figure 6F). In addition, ATP level in sh-Sun2 stable cell lines could be decreased by Si-SAA1 downregulation $(P<0.05)$ (Figure 6G), and cell proliferation also be inhibited by Si-SAA1 downregulation $(P<0.05)$ (Figure 6H). These results suggest that FAO,
A

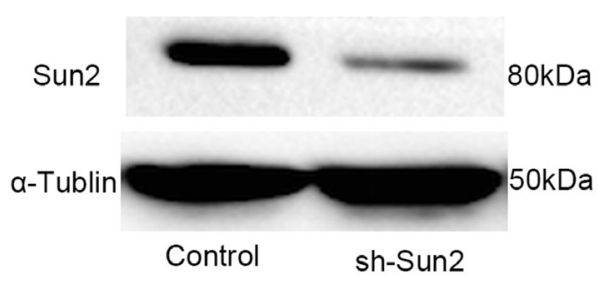

C

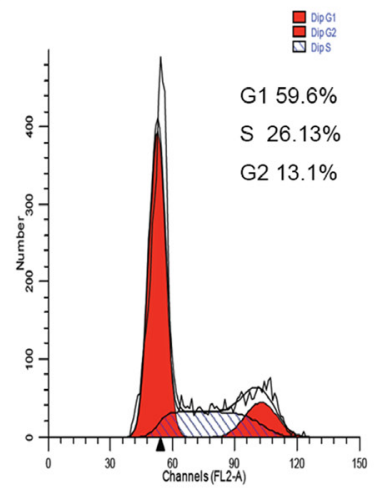

Control

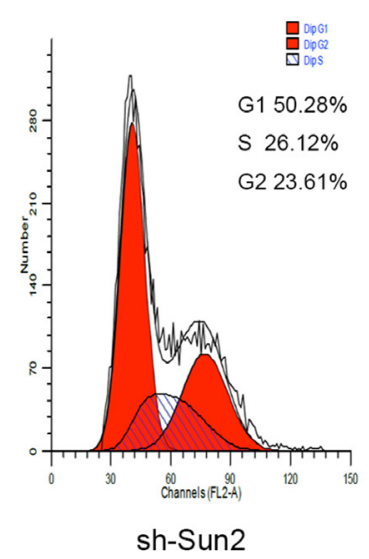

sh-Sun2
B

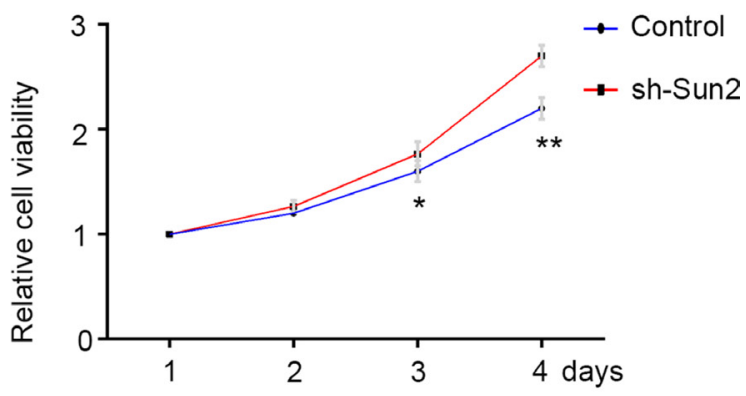

D
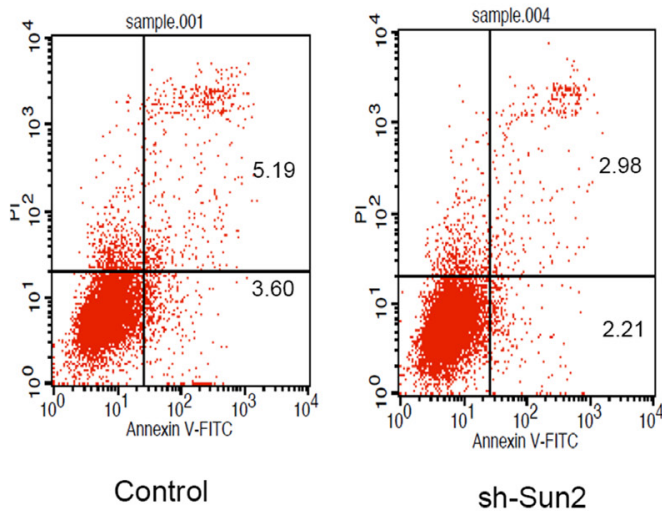

sh-Sun2

$E$
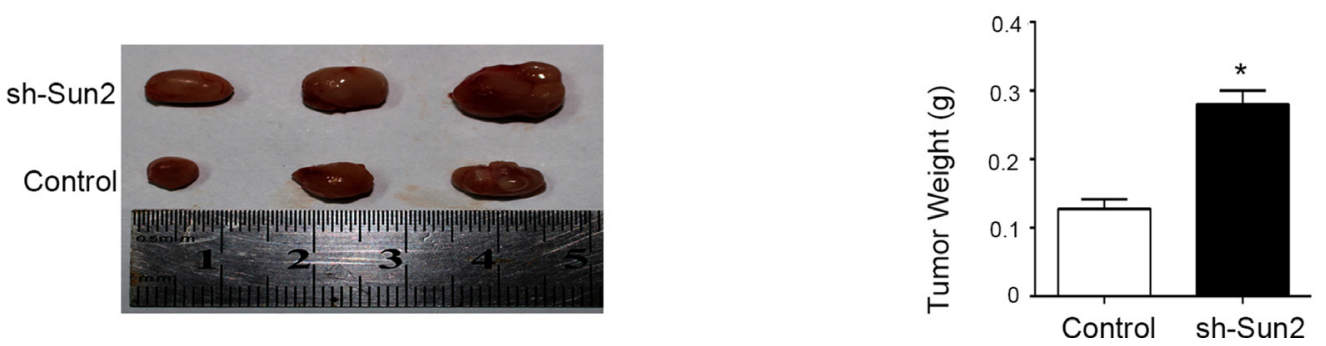

Figure 4: Sun2 knockdownpromotes the prostate cancer growth. (A) Western blot was used to confirm the sh-Sun2 transfection efficiency. (B) Cell proliferation levels were measured using the cell counting kit-8 at 1, 2, 3 and 4 days. (C) and (D) Cell-cycle distribution and cell apoptosis were analyzed by flow cytometry, respectively. (E) Tumorigenicity assay in nude mice was performed in Sh-Sun2 downexpression stable cell lines vs.control. ${ }^{*} P<0.05,{ }^{*} P P<0.01$. 
ATP level and cell growth promoted by Sun2 loss was partially suppressed by SAA1.

\section{DISCUSSION}

Recent studies [11-13] reported that Sun2 expression was downregulated in various cancers and also played a pivotal role in cellular biological pathways. The aim of the present study was to elucidate the expression and role of Sun2 in prostate cancer. We identified Sun2 as a gene that is down-expressed in prostate cancer tissues $v s$.normal tissues, and that low expression of Sun2 was significantly correlated with the high Gleason score, pT, Lymph nodal invasionand clinical pathological stage. Additionally, decreased Sun2 expression predicts poor survival in prostate cancer and wasan Independent predictor of OS. Next, Sun2 overexpression inhibited the prostate cancer growth, whileSun2 downregulation promoted the prostate cancer growth. These results point to an important role of Sun2 in prostate cancer progression, though the underlying molecular mechanism is unknown.
It is widely acknowledged that metabolic characteristics of elevated glucose uptake and lactate production occur in majority of tumor, is called the Warburg effect [15]. Recent reports revealed that aberrant expression or activation of gene promoted or inhibited the cancer initiation and progression by altering cancer cellular metabolism [16]. Sun2 plays a tumor suppressor role by inhibiting the Warburg effect in lung cancer as previously described. Here, we showed that low Sun2 expression promoted prostate cancer progression by enhancing FAObut had no significant impact on glycolysis. Prostate cancer, as a hormone-dependent cancer, grows slowly and is mainly dependent on lipid oxidation for fuel more than on aerobic glycolysis [17].This is probably the reason why positron emission tomography (PET) with radiotracer18 F-fluorodeoxyglucose does not work well in prostate cancer and has been limited in imaging of prostate cancer diagnosis and follow-up [18]. Though the mechanisms by which prostate cancer cells use lipids to their benefit are poorly understood, now, this still represents a novel avenue to investigate new nontoxic therapeutic approaches to prostate cancer treatment. Schlaepfer et al. [19]
A

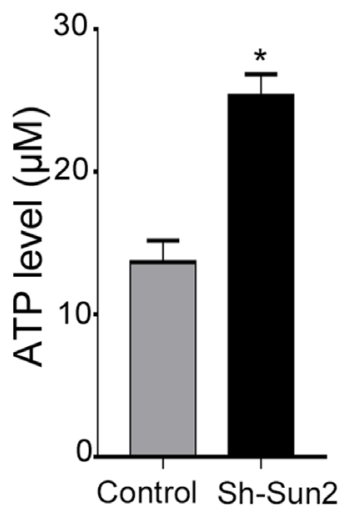

D

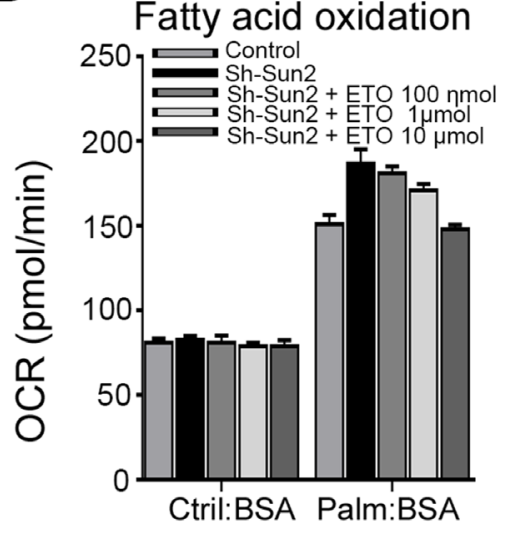

$\mathrm{B}$

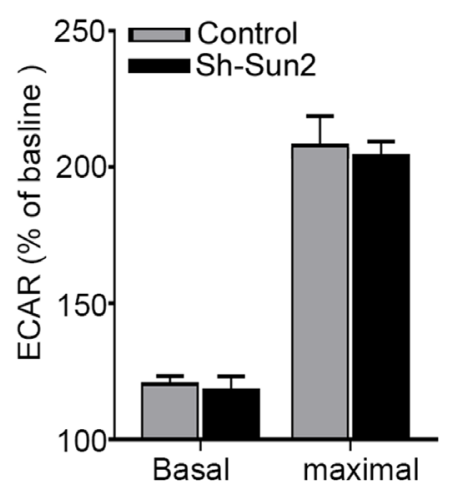

E

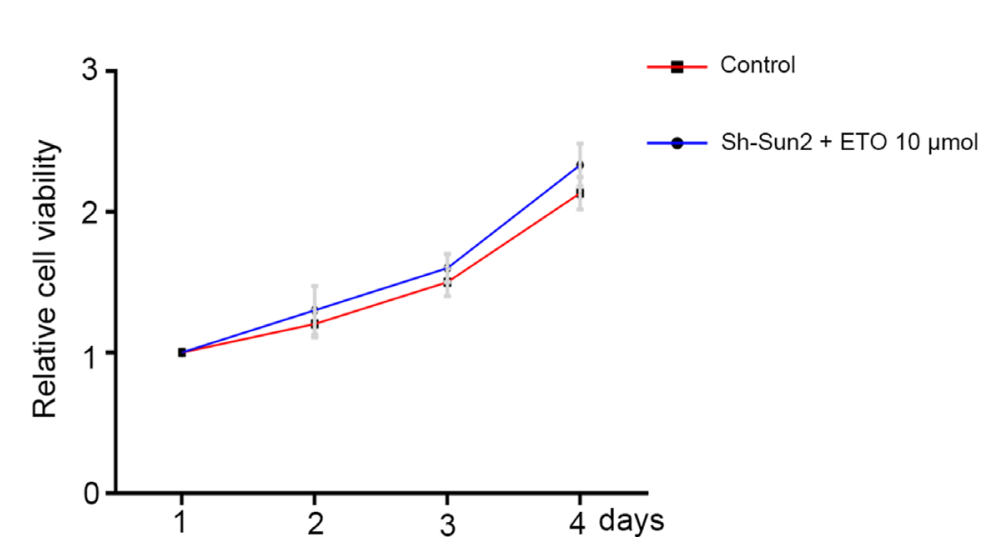

C Fatty acid oxidation

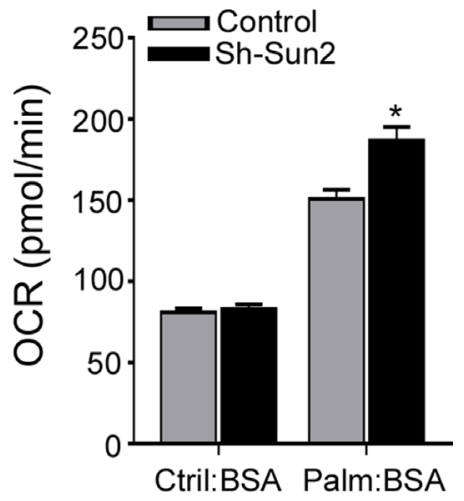

Ctril:BSA Palm:BSA

Figure 5: Sun2 suppresses the fatty acid oxidation (FAO) in prostate cancer. (A) ATP concentrations were measured in shSun 2 prostate cancer cell lines and control. (B) The glycolytic rate was measured in sh-Sun2 prostate cancer cell lines and control. (C) FAO was measured in sh-Sun2 prostate cancer cell lines and control. (D) sh-Sun2 prostate cancer cell lines were cultures with ETO, 100 nmol, 1 umol and 10 umol for $24 \mathrm{~h}$, and FAO was measured. (E) sh-Sun2 prostate cancer cell lines were cultured with ETO10 umol, and cell proliferation was measured by CCK assay. $* P<0.05, * * P<0.01$. 
A

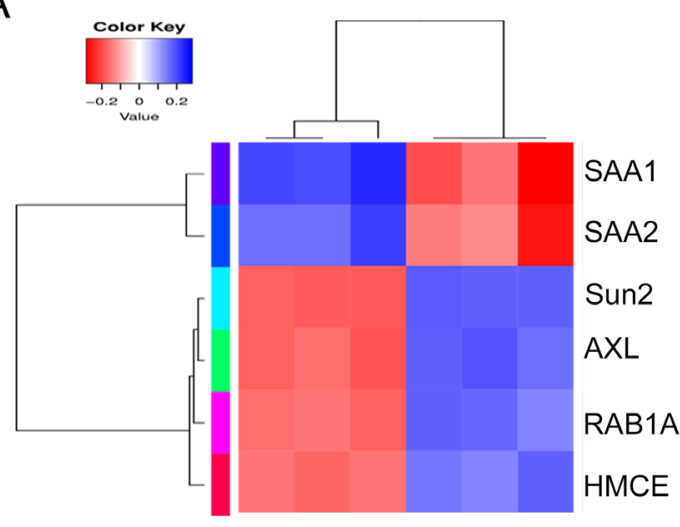

C

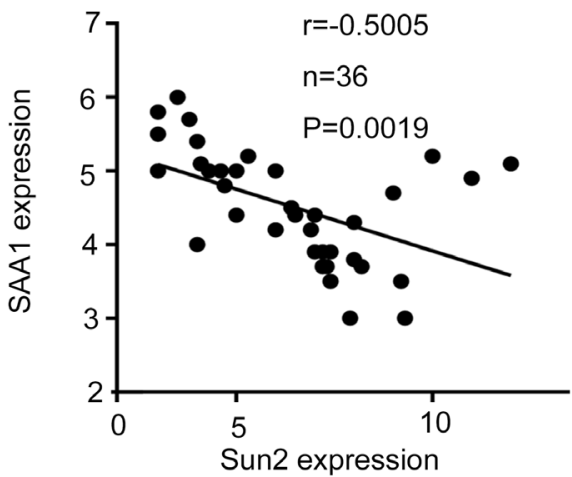

E

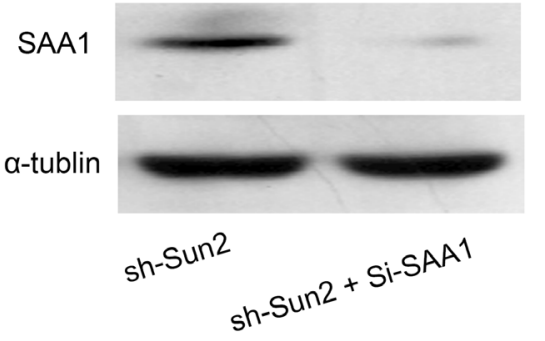

G

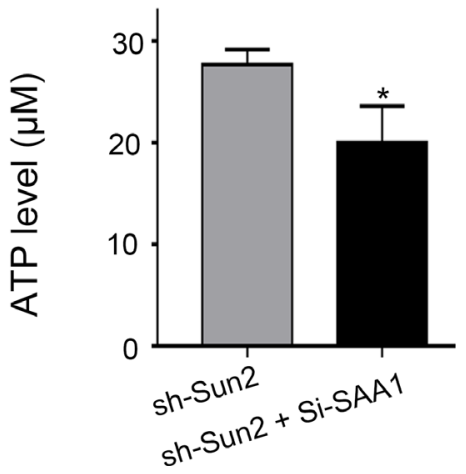

B

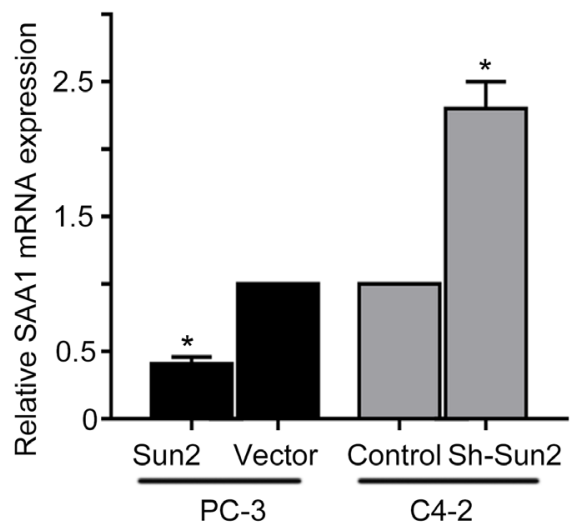

D

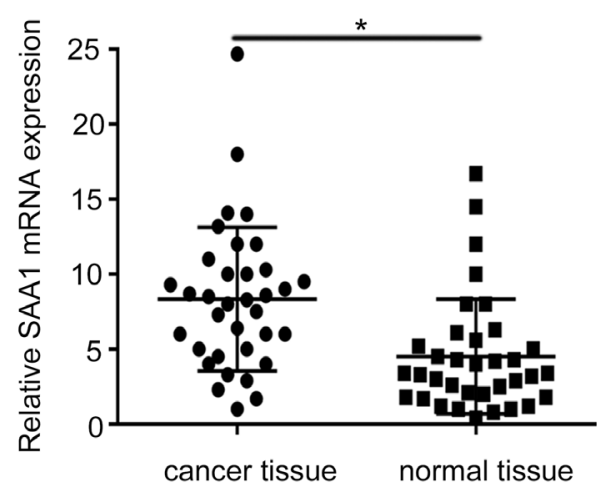

F

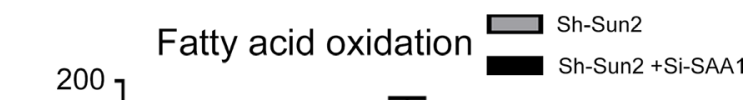

$\mathrm{H}$
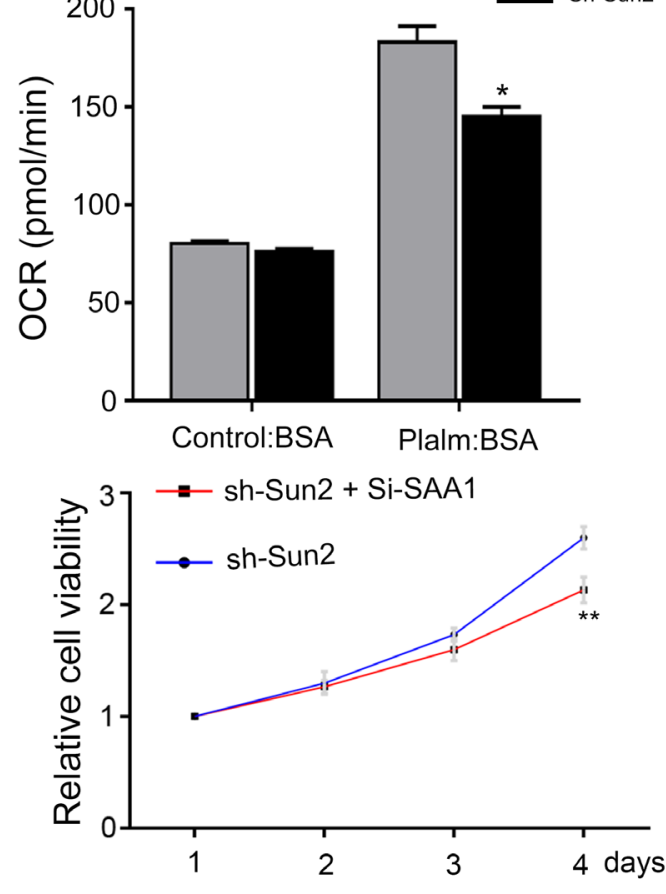

Figure 6: The underlying mechanism of FAO regulated by Sun 2 in prostate cancer. (A) Differentially expression genes of RNA in sh-Sun2 and control areshown in the form of a heat map. (B) SAA1 expression was evaluated in overexpression and knockdown of Sun 2 cell lines compared with control by RT-PCR analysis. (C) Correlation between Sun2 expression and SAA1 expression was observed in the prostate cancer tissues $(n=35)$. (D) Expression of SAA1 mRNA in prostate cancer tissues vs., matched adjacent normal tissues $(n=35)$. (E) Si-SAA transfection efficiency was identified by western blot. (F) FAO was evaluated in sh-Sun2 stable cell lines with Si-SAA transfection $v s$. Sun2 stable cell lines. (G) ATP level was evaluated in sh-Sun2 stable cell lines with Si-SAA transfection $v s$. Sun2 stable cell lines. (H) Cell viability was evaluated in sh-Sun2 stable cell lines with Si-SAA transfection $v s$. Sun 2 stable cell lines. ${ }^{*} P<0.05, * * P<0.01$. 
reported that Etomoxir, as a clinic drug for heart failure treatment, decreased prostate cancer growth by blocking lipid oxidation. Lloyd et al. [20] showed that peroxisomal enzyme, $\alpha$-methylacyl-CoA racemase (AMACR), which enhances the transformation of branched chain fatty acids to a form suitable for $\beta$-oxidation, were highly overexpressed in prostate cancer compared with normal prostate. Additionally, Lipid synthesis is increased in prostate cancer and Lipogenic enzyme, such as fatty acid synthase (FASN), ATP citrate lyase (ACLY) and acetylCoA carboxylase $\alpha$ (ACACA) is highly overexpressed in prostate cancer [21]. However, the mechanism of FAO regulated by Sun 2 in prostate cancer is unclear.

To explore the mechanism of FAO regulated by Sun2 in prostate cancer, six genes (SAA1, SAA2, AXL, RAB1A and HMCE) were identified by RNA sequence. We observed significant a significant inverse correlation between Sun2 and SAA1 in prostate cancer. And FAO, ATP level and cell growth increasing in sh-Sun2downexpressing prostate cancer cells could be reversed by SAA1 interference Serum amyloid A protein (SAA) is an apolipoprotein that can replace apolipoprotein A1 (apoA1) as the major apolipoprotein of high density lipoprotein (HDL). It is well recognized that SAA plays an important role in lipid metabolism, but how SAA impacts lipid metabolism remains incompletely understood. Cha $\mathrm{H}$ et al [22] reported that SAA increased the expression of CPT1 and reduced PPAR $\gamma$ expression to increase FA oxidation. These results suggested SAA1 plays an important role of FAO regulated by Sun2 in prostate cancer. However, how SAA1 expression regulated Sun 2 in PCa is unknown, and we will explore the potential mechanism in the next step.

In summary, Sun2 expression is reduced in prostate cancer, and may prove to be a useful predictor of poor clinical outcomes of prostate cancer patients. Additionally, loss of Sun 2 promotes the prostate cancer progression by enhancing FAO.

\section{MATERIALS AND METHODS}

\section{Ethics statement}

This experiment was approved by the institutional Ethical Committee of Shanghai Ninth Peoples' Hospital. All patients signed informed consent provided by our team before initiation of the experiment.

\section{Patient' specimens}

Prostate sample including adjacent non-neoplastic tissues and primary tumors were obtained from 90 patients with prostate cancer, whounderwent radical prostatectomy in Shanghai Ninth peoples' Hospital as the primary therapy (without hormonal or radiotherapy) between 2005 and 2010. 2010 pTNM AJCC classification and Gleason grading system were used to evaluate the tumors. In addition, no other relative treatment such as endocrinotherapy or radiotherapy were applied before surgery.

\section{Immunostaining and evaluation}

Immunohistochemical staining was performed with the primary antibody for Sun2 (ab124916, Abcam, UK). The procedure details for immunohistochemical staining and immunostaining was described in our previous study [22]. A low-level Sun2 expression was defined when the staining score in cancer tissues was less than or equal to that in adjacent normal tissues. And high level was defined when staining score in cancerous tissues displayed a higher than that in adjacent normal tissue.

\section{Cell culture}

Hormone-refractory prostate cancer cell lines, C4-2 and PC-3, were cultured in RPMI-1640 medium containing 10\% FBS (Gibco, USA) and $100 \mathrm{U} / \mathrm{ml}$ penicillin/streptomycin (Gibco, USA) at $37^{\circ} \mathrm{C}$ in $5 \% \mathrm{CO} 2$.

\section{Plasmids}

Full-length human Sun2 cDNA (gift from Dr. Zhaocai Zhou) was cloned into a pCDH-CMV-MCS-EF1puro vector, and oligonucleotides targeting Sun2 (shR NA 5'-GCAAGACTCAGAAGACCTCTT-3') [13] were synthesized and inserted into pLKO.1 vectors. All recombinant plasmids were verified through DNA sequencing.

\section{Cell transfection and stable cell lines}

The Sun2 expression or a control plasmid, the Sun2 targeting or scrambled shRNA plasmids were cotransfected with pMD.2G and PSPAX2 into HEK-293FT cells using Lipofectamine LTX (Invitrogen) according to the manufacturer's protocol. Viruses in the culture supernatants were harvested $48 \mathrm{~h}$ after transfection and infected cells two times at 24 -h intervals. After infection, cells were selected with puromycin.

\section{Quantitative real-time RT-PCR}

Total RNA was extracted from the fresh sample and cultured cells and quantified by qRT-PCR as described previously [10]. The primer sequences for amplification were Sun2, SAA1 and GAPDH [13, 14]. The PCR conditions were as follows: $94^{\circ} \mathrm{C}$ for $10 \mathrm{~min}$, then 40 cycles of $94^{\circ} \mathrm{C}$ for $30 \mathrm{sec}, 55-58^{\circ} \mathrm{C}$ for $30 \mathrm{sec}$, and $72^{\circ} \mathrm{C}$ for $45 \mathrm{sec}$, followed by $72^{\circ} \mathrm{C}$ for $10 \mathrm{~min}$. The quantity of each gene was normalized against GAPDH.

\section{Western-blot analysis}

Western-blot analysis was performed according to standard protocols as described previously [11]. In brief, 
membranes (PVDF membranes) were incubated with antibodies against Sun2 (ab124916, Abcam, UK), $\alpha$-tublin (H3663, Sigma Aldrich, USA) or SAA1 (ab201660, Abcam, USA).The intensities of light-emitting bands were checked and quantified using AmershamImager 600 (GE Healthcare,USA). $\alpha$-tublin was used as an internal control.

\section{Cell proliferation assay}

For cell proliferation assay, cells $\left(2 \times 10^{3}\right.$ cells/ well) were seeded in 96-well plates. Cell viability was determined using the Cell Counting Kit-8 (Sigma, China) according to manufacturer's instruction. Etomoxir (ETO) was purchased from Sigma (Sigma Aldrich, USA).

\section{Cell cycle and cell appotosis analysis}

For cell cycle assay, The cells were harvested and fixed in $70 \%$ ethanol at $4{ }^{\circ} \mathrm{C}$ for at least $24 \mathrm{~h}$. Cells were subsequently resuspended in PBS, incubated with propidium iodide (PI) (Abcam, USA) in the presence of RNase A for $30 \mathrm{~min}$ in the dark, and finally subjected to flow cytometry analysis. For cell apoptosis analysis, cells were harvested and stained with PI and annexin V-FITC (Abcam, USA), incubated at room temperature in the dark for $15 \mathrm{~min}$, and finally subjected to flow cytometry analysis.

\section{ATP measurements}

The ATP determination kit (Beyotime Biotechnology, China) was used to evaluate the ATP measurements. The ATP concentration was determined based on comparison with a concurrent standard curve.

\section{The glycolytic rate and fatty acid oxidation assay}

The extracellular acidification rate (ECAR) and Oxygen consumption rate (OCR) were used to evaluate the capacity of the glycolytic rate and FAO respectively by the Seahorse XF96 extracellular flux analyzer. Briefly, $2 \times 10^{4}$ cells per well were seeded overnight in XF96 well plates. For Glycolytic rate assay, One hour before XF assay, cells were equilibrated with unbuffered DMEM and maintained at $37^{\circ} \mathrm{C}$ for $\mathrm{PH}$ stabilization. Analyses were performed both at basal conditions and after injection of OLI (1 mg/ml), FCCP (1 mM) and Antimycin A (5 mM). For Fatty acid oxidation assay, the FAO assay KHB buffer supplemented with $2.5 \mathrm{mM}$ glucose, $0.5 \mathrm{mM}$ carnitine and $5 \mathrm{mM}$ HEPES was added and adjusted to $\mathrm{pH} 7.4$ in a $37^{\circ} \mathrm{C}$ incubator. To examine free fatty acid oxidation, BSA conjugated palmitate (Seahorse Bioscience) was added to a final concentration of $50 \mu \mathrm{M}$. Basal OCR of cells treated with Palmitate-BSA or BSA vehicle alone was measured.

\section{Tumorigenicity assay in nude mice}

The cells were resuspended in a solution of Matrigel (BD, USA) and PBS (equal volume), and then injected subcutaneously into the flanks of 8 -week-old male $\mathrm{BALB} / \mathrm{c}$ nude mice at $3 \times 10^{6}$ cells per site. To evaluate the tumor formation abilities of cells transfected with Sun 2 plasmid or Sun 2 shRNA, all mices were sacrificed in 5 weeks and the tumor were weighed.

\section{RNA sequencing by Illumina HiSeq}

In beif, total RNA of each sample was extracted using TRIzol Reagent (Invitrogen)/RNeasy Mini Kit (Qiagen)/other kits. Total RNA of each sample was quantified and qualified by Agilent 2100 Bioanalyzer (Agilent Technologies, Palo Alto, CA, USA), and 1\% agrose gel. $1 \mu \mathrm{g}$ total RNA with RIN value above 7 was used for following library preparation. Next generation sequencing library preparations were constructed according to the manufacturer's protocol $\left(\mathrm{NEBNext}^{\circledR}\right.$ Ultra $^{\mathrm{TM}}$ RNA Library Prep Kit for Illumina $\left.{ }^{\circledR}\right)$.Sequencing was carried out using a $2 \times 150$ bp paired-end $(\mathrm{PE})$ configuration; image analysis and base calling were conducted by the HiSeq Control Software (HCS) + OLB + GAPipeline-1.6 (Illumina) on the HiSeq instrument. The sequences were processed and analyzed by GENEWIZ INC (Suzhou, China).

\section{Statistical analysis}

Data are expressed as mean $\pm \mathrm{SD}$. Differences in mRNA detection and $\mathrm{H}$-score between prostate cancer and self-paired normal tissues were assessed by paired $t$-test. The chi-squared test was used to assess the correlation between Sun 2 expression and patient clinicopathological characteristics.Kaplan-Meier method and log-rank tests were performed to assess the survival rate and compare differences in survival curves. Stepwise Cox proportional hazard models were used to analyze independent predictors associated with overall survival (OS).Others were analyzed using Student's $t$-test. A value of $P<0.05$ was considered to be significant.

\section{Abbreviations}

overall survival (OS), fatty acid oxidation (FAO), fatty acid synthase (FASN), ATP citrate lyase (ACLY) and acetyl-CoA carboxylase $\alpha$ (ACACA), apolipoprotein A1 (apoA1), high density lipoprotein (HDL), $\alpha$-methylacylCoA racemase (AMACR), positron emission tomography (PET), Serum prostate-specific antigen (PSA), Serum amyloid A1 (SAA1), Serum amyloid A2 (SAA2), receptor tyrosine kinase (AXL), Ras-related protein Rab-1A (RAB1A), Etomoxir (ETO), Nuclear envelope (NE), Outer inner nuclear (ONM), Inner nuclear membrane (INM), 
perinuclear space (PNS), LINC (linker of nucleoskeleton and cytoskeleton), postoperative T stage (pT).

\section{Authors' contributions}

$\mathrm{Xu}$ Bin, Chen Jia and Wang Zhong designed the research; Cheng Yajun, Ye Chen and Li Xiaosa performed the research; Wang Xiao contributed new reagents or analytic tools; Cheng yajun and Chen Jia analyzed the data and prepared the figures; Cheng Yajun and Xu Bin drafted the manuscript; all authors have reviewed the manuscript.

\section{ACKNOWLEDGMENTS}

This work is supported by the National Natural Science Foundation of China (No.81472398).

\section{CONFLICTS OF INTEREST}

The authors have no conflicts of interest to disclose.

\section{REFERENCES}

1. Chen W, Zheng R, Baade PD, Zhang S, Zeng H, Bray F, Jemal A, Yu XQ, He J. Cancer statistics in China, 2015. CA Cancer J Clin. 2016; 66:115-32.

2. Ferlay J, Soerjomataram I, Dikshit R, Eser S, Mathers C, Rebelo M, Parkin DM, Forman D, Bray F. Cancer incidence and mortality worldwide: sources, methods and major patterns in GLOBOCAN 2012. Int J Cancer. 2015; 136:359-86.

3. Zhu X, Albertsen PC, Andriole GL, Roobol MJ, Schröder FH, Vickers AJ. Riskbased prostate cancer screening. Eur Urol. 2012; 61:652-61.

4. Ferlay J, Shin HR, Bray F, Forman D, Mathers C, Parkin DM. Estimates of worldwide burden of cancer in 2008: GLOBOCAN 2008. Int J Cancer. 2010; 127:2893-917.

5. Xu S, Yi XM, Zhou WQ, Cheng W, Ge JP, Zhang ZY. Downregulation of miR-129 in peripheral blood mononuclear cells is a diagnostic and prognostic biomarker in prostate cancer. Int J Clin Exp Pathol. 2015; 8:1433514344

6. Cuzick J, Thorat MA, Andriole G, Brawley OW, Brown PH, Culig Z, Eeles RA, Ford LG, Hamdy FC, Holmberg L, Ilic D, Key TJ, La Vecchia C, et al. Prevention and early detection of prostate cancer. Lancet Oncol. 2014; 15:e484-92.

7. Shimi T, Butin-Israeli V, Goldman RD. The functions of the nuclear envelope in mediating the molecular crosstalk between the nucleus and the cytoplasm. Curr Opin Cell Biol. 2012; 24:71-8.

8. Maraldi NM, Lattanzi G, Cenni V, Bavelloni A, Marmiroli S, Manzoli FA. Laminopathies and A-type lamin-associated signalling pathways. Adv Enzyme Regul. 2010; 50:248-61.
9. Isermann $\mathrm{P}$, Lammerding J. Nuclear mechanics and mechanotransduction in health and disease. Curr Biol. 2013; 23:R1113-R1121.

10. Donahue DA, Amraoui S, di Nunzio F, Kieffer C, Porrot F, Opp S, Diaz-Griffero F, Casartelli N, Schwartz O. SUN2 Overexpression Deforms Nuclear Shape and Inhibits HIV. J Virol. 2016; 90:4199-214.

11. Hsieh TH, Chien CL, Lee YH, Lin CI, Hsieh JY, Chao ME, Liu DJ, Chu SS, Chen W, Lin SC, Ho DM, Liu RS, Lin CH, et al. Downregulation of SUN2, a novel tumor suppressor, mediates miR-221/222-induced malignancy in central nervous system embryonal tumors. Carcinogenesis. 2014; $35: 2164-2174$.

12. Matsumoto A, Hieda M, Yokoyama Y, Nishioka Y, Yoshidome K, Tsujimoto M, Matsuura N. Global loss of a nuclear lamina component, lamin $\mathrm{A} / \mathrm{C}$, and LINC complex components SUN1, SUN2, and nesprin-2 in breast cancer. Cancer Med. 2015; 4:1547-1557.

13. Lv XB, Liu L, Cheng C, Yu B, Xiong L, Hu K, Tang J, Zeng L, Sang Y. SUN2 exerts tumor suppressor functions by suppressing the Warburg effect in lung cancer. Sci Rep. 2015; 10:17940.

14. Chen $\mathrm{CH}$, Wang PH, Liu BH, Hsu HH, Mersmann HJ, Ding ST. Serum amyloid A protein regulates the expression of porcine genes related to lipid metabolism. J Nutr. 2008; 138:674-9.

15. Warburg O. On the origin of cancer cells. Science. 1956; 123:309-314.

16. Schlaepfer IR, Rider L, Rodrigues LU, Gijón MA, Pac CT, Romero L, Cimic A, Sirintrapun SJ, Glodé LM, Eckel RH, Cramer SD. Lipid catabolism via CPT1 as a therapeutic target for prostate cancer. Mol Cancer Ther. 2014; 13:2361-71.

17. Vander Heiden MG, Cantley LC, Thompson CB. Understanding the Warburg effect: the metabolic requirements of cell proliferation. Science. 2009; 324:1029-1033.

18. Kitajima K, Murphy RC, Nathan MA, Sugimura K. Update on positron emission tomography for imaging of prostate cancer. Int J Urol. 2014; 21:12-23.

19. Deep G, Schlaepfer IR. Aberrant Lipid Metabolism Promotes Prostate Cancer: Role in Cell Survival under Hypoxia and Extracellular Vesicles Biogenesis. Int J Mol Sci. 2016; 17.

20. Lloyd MD, Yevglevskis M, Lee GL, Wood PJ, Threadgill MD, Woodman TJ. $\alpha$-Methylacyl-CoA racemase (AMACR): Metabolic enzyme, drug metabolizer and cancer marker P504S. Prog Lipid Res. 2013; 52:220-230.

21. Wu X, Daniels G, Lee P, Monaco ME. Lipid metabolism in prostate cancer. Am J Clin Exp Urol. 2014; 2:111-20.

22. Wei ZR, Liang C, Feng D, Cheng YJ, Wang WM, Yang DJ, Wang YX, Cai QP. Low tristetraprolin expression promotes cell proliferation and predicts poor patients outcome in pancreatic cancer. Oncotarget. 2016; 7:17737-17750. https://doi.org/10.18632/oncotarget.7397. 